\title{
What is the optimal adjuvant therapy for T3NO lung cancer invading the chest wall?
}

\author{
Makoto Suzuki, Takeshi Mori, Kenji Shiraishi, Koei Ikeda, Yoshiko Masuda, Eri Matsubara, Chika \\ Shirakami, Hironori Hinokuma
}

Department of Thoracic Surgery, Kumamoto University Hospital, Kumamoto, Japan

Correspondence to: Makoto Suzuki, MD, PhD. Department of Thoracic Surgery, Kumamoto University Hospital, 1-1-1 Honjo, Chuo-ku, Kumamoto 860-8556, Japan. Email: smakoto@kumamoto-u.ac.jp.

Provenance: This is an invited Editorial commissioned by Section Editor Dr. Dong Wang (Department of Thoracic Surgery, Shandong Provincial Hospital Affiliated to Shandong University, Jinan, China).

Comment on: Gao SJ, Corso CD, Blasberg JD, et al. Role of Adjuvant Therapy for Node-Negative Lung Cancer Invading the Chest Wall. Clin Lung Cancer 2017;18:169-77.e4.

Submitted Sep 24, 2017. Accepted for publication Oct 04, 2017.

doi: $10.21037 /$ jtd.2017.10.73

View this article at: http://dx.doi.org/10.21037/jtd.2017.10.73

Even after complete removal of stage I-III non-small cell lung cancer (NSCLC), relapse is common. Adjuvant treatment has been the focus of efforts to improve overall survival (OS) of patients who undergo complete resection of stage I-III NSCLC.

Recently, the role and efficacy of adjuvant chemotherapy for stage II-IIIA NSCLC was established by the LACE in 2008 (1). Postoperative cisplatin-based chemotherapy significantly improved survival in patients with NSCLC, with a 5 -year absolute benefit of $5.4 \%$ from chemotherapy. The benefits of adjuvant chemotherapy were shown in patients with stage II-III disease but not in patients with stage I disease. There was a possibility of more harm in patients with stage IA disease.

In the last decade, the longer-term impact of adjuvant chemotherapy or other adjuvant therapies such as EGFRTKIs and immunotherapy for resected NSCLC has been reported. The American Society of Clinical Oncology (ASCO) guidelines on adjuvant therapy for resected NSCLC were updated in April 2017 (2). In summary, cisplatin-based adjuvant chemotherapy is recommended for stage IIA/B and IIIA disease. Patients with stage IB should be evaluated to assess the risks and benefits of adjuvant chemotherapy. Adjuvant radiation therapy is not recommended for stage IA/B and IIA/B disease. Patients with stage IIIA (N2) disease should be evaluated to assess the risks and benefits of adjuvant radiation therapy.
In addition, a systematic review of the role of systemic adjuvant therapy in completely resected NSCLC demonstrated that cytotoxic adjuvant chemotherapy improved OS in patients with resected stage II-III NSCLC (3). On the other hand, T3N0 NSCLC tumors with chest wall invasion may need a different approach. Stoelben and Ludwig reported that only approximately $5 \%$ of all patients who undergo surgical resection for lung cancer present with chest wall invasion and the OS of T3N0M0 tumors reaches 40-50\% (4).

In those situations, the study by Gao et al. is the first large retrospective analysis to examine the outcomes of postoperative treatment modalities for T3N0 NSCLC with chest wall invasion (5). Their cohort was stratified by margin status (positive or negative) and tumor size ( $\geq 4$ or $\leq 4 \mathrm{~cm}$ ). Subgroup analysis of margin status was performed because the NCCN guidelines suggest that margin status should be a determinant of adjuvant therapy use. Subgroup analysis of tumor size was performed because the CALGB 9633 study showed that adjuvant chemotherapy conferred a significant survival advantage on patients with tumors $\geq 4 \mathrm{~cm}$ (6). In that study, 319 patients received surgery alone, 177 patients received surgery followed by chemotherapy, 93 patients received surgery followed by radiotherapy, and 170 patients received surgery followed by chemoradiation. Because of the retrospective nature of the study, there was a higher proportion of elderly patients in the surgery alone group compared with other groups. In addition, 
margin-positive patients received radiotherapy more often than other types of treatment. As a result, patients with tumors $>4 \mathrm{~cm}$ benefited from chemoradiation and patients with tumors $<4 \mathrm{~cm}$ benefited only from chemotherapy. Margin-negative patients with tumors $>4 \mathrm{~cm}$ benefited from chemoradiation, and margin-positive patients with tumors $>4 \mathrm{~cm}$ benefited from either chemoradiation or radiotherapy alone.

First, we should note that studies evaluating adjuvant therapy for patients with T3N0 NSCLC who underwent chest wall resection like the study by Gao $e t a l$. are very rare. As a result, standard treatments are undefined and many modalities, such as a combination of surgery, chemotherapy, and radiotherapy, are often used clinically.

Ahmad et al. reported that adjuvant chemotherapy after lobectomy with chest wall resection in pathologic T3N0 stage II disease is associated with improved OS in an analysis of data from the National Cancer Database (NCDB) (7). In addition, we can refer to the NCCN guidelines (8). According to the NCCN guidelines for resected T3N0 NSCLC, chemotherapy is recommended for negative margins and re-resection plus chemotherapy or chemoradiation is recommended for positive margins.

Small but distinct differences between the study by Gao et al. and the analysis of NCDB data and NCCN guidelines exist. A novel finding in the study by Gao et al. is that when tumor size was stratified, patients with tumors $>4 \mathrm{~cm}$ experienced a significant increase in OS when receiving adjuvant chemoradiation, regardless of margin status. However, the analysis of NCDB data and the NCCN guidelines recommend only chemotherapy when margins are negative.

We do not have the correct answer and evidence on this topic. However, we can turn to the effectiveness of postoperative radiotherapy (PORT) in stage III-N2 patients with completely resected NSCLC in improving OS. The benefit of PORT for these patients had been controversial $(9,10)$ until the results of the Lung ART study $(11)$. However, a recent retrospective study and meta-analysis of PORT demonstrated that it had a positive effect $(12,13)$.

PORT for stage III-N2 completely resected NSCLC and adjuvant chemoradiation for margin-negative pathologic T3N0 NSCLC tumors $>4 \mathrm{~cm}$ invading the chest wall are similar in that there are no visible tumors in the roentgen images of the radiation fields. Considering the possible positive effect of PORT on OS in stage III-N2 patients with completely resected NSCLC, chemotherapy plus radiation might also have a positive effect on OS in patients with margin-negative pathologic T3N0 NSCLC tumors $>4 \mathrm{~cm}$ who undergo chest wall resection.

Further prospective clinical investigation is needed to clarify whether chemotherapy or chemoradiation is the best adjuvant treatment for tumors $>4 \mathrm{~cm}$ with negative margins. Although their study is retrospective and there were some imbalances in the clinicopathologic features of the study patients, their results encourage us to develop personalized treatment strategies for pathologic T3N0 NSCLC with chest wall invasion.

\section{Acknowledgements}

Funding: This work was partially supported by a Grant-inAid for Scientific Research from the Ministry of Education, Science, Sports, Culture and Technology of Japan (17K10795).

\section{Footnote}

Conflicts of Interest: The authors have no conflicts of interest to declare.

\section{References}

1. Pignon JP, Tribodet H, Scagliotti GV, et al. Lung adjuvant cisplatin evaluation: a pooled analysis by the LACE Collaborative Group. J Clin Oncol 2008;26:3552-9.

2. Kris MG, Gaspar LE, Chaft JE, et al. Adjuvant Systemic Therapy and Adjuvant Radiation Therapy for Stage I to IIIA Completely Resected Non-Small-Cell Lung Cancers: American Society of Clinical Oncology/Cancer Care Ontario Clinical Practice Guideline Update. J Clin Oncol 2017;35:2960-74.

3. Bradbury P, Sivajohanathan D, Chan A, et al. Postoperative Adjuvant Systemic Therapy in Completely Resected NonSmall-Cell Lung Cancer: A Systematic Review. Clin Lung Cancer 2017;18:259-73.e8.

4. Stoelben E, Ludwig C. Chest wall resection for lung cancer: indications and techniques. Eur J Cardiothorac Surg 2009;35:450-6.

5. Gao SJ, Corso CD, Blasberg JD, et al. Role of Adjuvant Therapy for Node-Negative Lung Cancer Invading the Chest Wall. Clin Lung Cancer 2017;18:169-77.e4.

6. Strauss GM, Herndon JE 2nd, Maddaus MA, et al. Adjuvant paclitaxel plus carboplatin compared with observation in stage IB non-small-cell lung cancer: CALGB 9633 with the Cancer and Leukemia Group B, 
Radiation Therapy Oncology Group, and North Central Cancer Treatment Group Study Groups. J Clin Oncol 2008;26:5043-51.

7. Ahmad U, Crabtree TD, Patel AP, et al. Adjuvant Chemotherapy Is Associated With Improved Survival in Locally Invasive Node Negative Non-Small Cell Lung Cancer. Ann Thorac Surg 2017;104:303-7.

8. Ettinger DS, Wood DE, Akerley W, et al. Non-Small Cell Lung Cancer, Version 6.2015. J Natl Compr Canc Netw 2015;13:515-24.

9. Burdett S, Rydzewska L, Tierney J, et al. Postoperative radiotherapy for non-small cell lung cancer. Cochrane Database Syst Rev 2016;10:CD002142.

10. Zhang S, Sun X, Sun L, et al. Benefits of postoperative thoracic radiotherapy for small cell lung cancer subdivided

Cite this article as: Suzuki M, Mori T, Shiraishi K, Ikeda K, Masuda Y, Matsubara E, Shirakami C, Hinokuma H. What is the optimal adjuvant therapy for T3N0 lung cancer invading the chest wall? J Thorac Dis 2017;9(11):4233-4235. doi: 10.21037/jtd.2017.10.73 by lymph node stage: a systematic review and metaanalysis. J Thorac Dis 2017;9:1257-64.

11. Le Péchoux C, Dunant A, Pignon JP, et al. Need for a new trial to evaluate adjuvant postoperative radiotherapy in non-small-cell lung cancer patients with N2 mediastinal involvement. J Clin Oncol 2007;25:e10-1.

12. Billiet C, Decaluwe H, Peeters S, et al. Modern postoperative radiotherapy for stage III non-small cell lung cancer may improve local control and survival: a metaanalysis. Radiother Oncol 2014;110:3-8.

13. Robinson CG, Patel AP, Bradley JD, et al. Postoperative radiotherapy for pathologic N2 non-small-cell lung cancer treated with adjuvant chemotherapy: a review of the National Cancer Data Base. J Clin Oncol 2015;33:870-6. 